\title{
Magnetic Contactless Crank-rocker Machine
}

\author{
Adnan Ishtay ${ }^{1}$, Mohammad Awwad Al-Dabbas ${ }^{2 *}$ \\ ${ }^{1}$ Design and Development Department, Smart Measure Company, Riyadh, Saudi Arabia. \\ ${ }^{2}$ Mechanical Engineering Department, Mutah University P.O. Box 7, Mutah, Karak 61710, Jordan.
}

\begin{abstract}
Objective: In this paper, a proposed technique of motion transmission is introduced, which is based on the crank-rocker principle of motion. The energy transmission action is performed through magnetic force, in which no direct connection is made between the energy source input and the energy load output. Also, to illustrate the concept of motion and to approve the continuity of energy transmission using this proposed technique, a simple model of this mechanism has been built and run, showing the basic sequence of operation. Methodology/analysis: In this mechanical transmission mechanism, one side is rotating and the other side is vibrating, in which any side is energy input (which is usually the vibrating rocker), and the other side is energy output (which is the rotating crank). That seems similar to the classical crank-rocker machine in the four-bar mechanism, but without direct mechanical contact between the input and output energy stream. The concept of motion and mathematical analysis with structuring conditions is provided in this paper, where the dynamic analysis of the system is left for future work. A pilot physical prototype is manufactured and experimentally tested, validating the proposed design. Findings: The structural parameters of this proposed contactless crank-rocker machine have been modelled and simulated using the MATLAB program. It shows that these parameters could be selected and optimized to guarantee the minimum conditions for continued energy transmission. Based on these parameters, a simple model has been built and operated, which illustrates the concept of motion and validates the finding of MATLAB simulation. Novelty/improvement: Contactless crank-rocker motion is a very promising technique. It is possible to apply it in many applications, like the energy harvesting area, and it could be employed certainly in specific designs, such as MEMS, where no other motion transmission types can be used.
\end{abstract}

\section{Keywords:}

Motion Transmission;

Contactless Motion;

Magnetic Force Applications;

Energy Harvesting;

Motor.

Article History:

$\begin{array}{llll}\text { Received: } & 08 & \text { October } & 2021 \\ \text { Revised: } & 21 & \text { December } & 2021 \\ \text { Accepted: } & 03 & \text { January } & 2022 \\ \text { Available online: } & 27 & \text { February } & 2022\end{array}$

\section{1- Introduction}

The proposed mechanism in this research presents a way of power converting approach, which could be used mainly in energy harvesting of vibrating energy sources or to transfer mechanical energy indirectly to loads without direct contact in special applications. Unlike most of the current contact-less energy transfer applications that use electrical media in the transferring process [1], the proposed mechanism in this research uses passive permanent magnets to transfer mechanical energy directly $[2,3]$. Several research studies have been performed on the design and analysis of harvesting the vibration energy from different architectures with the goal of optimizing the output power. In mechanical energy transmission, friction losses represent the main type of energy loss. It does not only minimize the transmitted useful energy but also increases the wear, heating, noise, etc. However, by developing a contactless effective energy transmission, the friction loss will be eliminated or minimized, which provides an efficient model, especially where the transferred energy is very small, as in MEMS applications [4, 5]. Over the last few years, more and more sophisticated methods of contactless energy transmission have been researched and produced. In the future, we'll be able to power our portable gadgets without having to connect them to anything, like cords and switches [6].

*CONTACT: madabbas@mutah.edu.jo

DOI: http://dx.doi.org/10.28991/ESJ-2022-06-02-07

(C) 2022 by the authors. Licensee ESJ, Italy. This is an open access article under the terms and conditions of the Creative Commons Attribution (CC-BY) license (https://creativecommons.org/licenses/by/4.0/). 
Many methods are utilized for the design of contactless energy transfer systems, and they may be categorized according to the mechanisms used for energy transmission between the transmitter and the receiver [1-4]. These devices may be based on acoustics, light, capacitive, or inductively linked. This latter one is essential in EV, as will be described in the next section [6]. In contrast to the classical crank rocker mechanism, which is one setup of a four-bar mechanism, a mechanism might be considered as a power transmitter and a motion transformer. The four-bar mechanism is commonly utilized in many mechanical engineering aspects. These planar mechanisms' primary parts are joints, links, or pairs that meet the criteria for many actual engineering purposes. The four-bar crank-rocker mechanism is the most commonly used type of planar mechanism in mechanical systems and devices. Furthermore, an essential factor in the planar mechanism's practical design is its transmission angle. The planar four-bar mechanism's transmission angle is the one between the output and the coupler links [7,8]. The provided mechanism consists of a rotary disk (crank), a strong magnet distributed evenly (at angles) around this disk, but with a steady reduction in polar length, up to a complete circle around the disk. A rocker arm moves along the diametrical axes of the disk, and a strong magnet is fixed on the rocker arm end on the same crank's magnet surface with the same direction of polarity as shown in Figure 1.

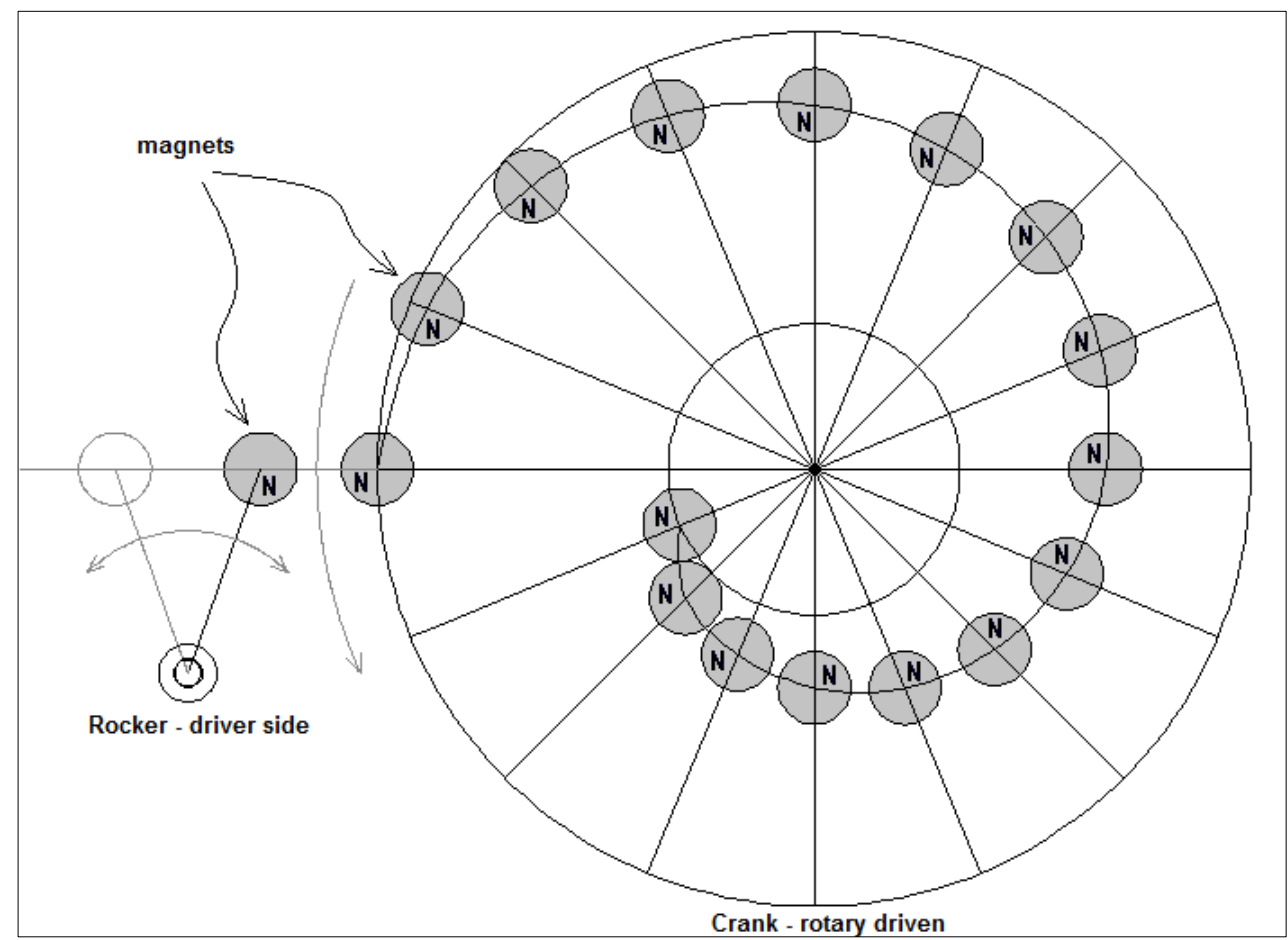

Figure 1. Scheme of proposed magnetic contactless crank-rocker machine

By moving the rocker's magnet (driver side) toward the disk, a repulsion force tries to move the crank (rotary driven disk) rotationally to reduce the impact of the repulsion force. The magnitude and direction of rotation strictly relate to the position of the rocker and the angle of rotation of the disk. By controlling and synchronizing the position of the rocker with the angle of the disk (this could be done by controlling the loading torque on the driven disk), continued and smooth power transmission could be moved from the rocker to the disk without any contact or mechanical link; this is the reason why we call this concept a "contactless" machine.

Energy harvesting of vibrating sources is one of the most favorable applications for such a contactless mechanism. For such an application, piezoelectric generators are usually used to retrieve energy [9, 10]. A contactless rocker machine can fit this application, where the vibrating source actuates the rocker and the rotary disk is driven indirectly to drive the rotary load. Micro magnets could also be used in MEMS, which could be a good use for them. In this case, the isolation is very important, and the small size improves the magnetic transfer of energy, which could be used in sensors.

\section{2- Literature Review}

Although different studies share some features with the provided technique in this work and have similar final functions, no one contains the same architecture and process of work. A mathematical dynamic analysis has been provided for two series of permanent magnets [11]. Based on nonlinear oscillators, a simple cantilever that composites electric, mechanic, and magneto with a tri-layered of long bar bidomain LN- metglas/steel spring and a large tip of permanent magnet is illustrated by Vidal et al. (2020) [12], which is trying to optimize its resonant performance against load impedance to get the best results from harvesting the energy from low frequency random vibration. 
A study of energy harvesting of ultra-low frequency using bi-stable motion and rotary-translational in a magnettethered oscillator is provided by $\mathrm{Fu}$ et al. (2020) [13]. A theoretical and experimental study is provided for design guidelines and analysis of magnetic springs used in vibration energy systems [14]. Redder et al. depict the contactless energy transmission unit. The author notes that such a system may be used without electrical connection or physical touch through a low-conductivity nonmagnetic medium [15]. El-Refaie et al. (2017) [16] present a different topology for rotary transformers, where large leakage inductances may be significantly reduced. This suggested rotating transformer has a lightweight, robust, non-magnetic, and non-conductive combination of non-ferrite rotating components, as well as a wide air gap, enabling it to operate safely at high speeds such as rotation. The fact that this innovative rotary transposal is possible makes it possible to be used for rotor-spindle synchronism devices for hybrid or electric vehicle traction. Utilizing a three-dimensional finite element technique, Kyung Woo has developed a unique calculation procedure for the contact-less energy transfer device's magnetic coupling coefficient in terms of primary and secondary core locations. The magnetic coupling coefficients are computed. The resonant circuit is used to calculate the currents and voltages at the secondary core locations, and these values are compared [17].

To realize his goal of enabling the robot to do a wide range of functions, Komiyama and Uchimura (2013) explain that a magnetic gear is applied to the robot to get a controller with multi-functionalities: positioning, force control, and velocity control. An approximation concept of cogging torque is implemented [18]. To make new implementations of such machines possible, Andrea Tilli and others present a unique control approach for doubly-fed induction units. The provided method makes it possible to use direct-drive versions of Doubly-Fed Induction Machines to move and feed the spinning portion where the rotor-side converter must be housed independently [19]. Mohammed et al. (2019) create a new crank-rocker engine that converts the oscillating motion of the curve-piston to the rotary motion of the crankshaft in order to determine the properties of the combusted fuel. A single curved-cylinder spark-ignition crank-rocker engine is described with a basic thermodynamic model [20]. Koo et al. (2002) designed and simulated a contactless energy transmission system for linear servo motor [21]. Sang et al. (2015) converted wave energy into electricity at a relatively high efficiency using a slider-crank wave energy converter [22]. Boeij (2009) Multi-Level Contactless Motion System In machine design, the acceleration and velocity of moving parts are often constrained by mechanical bearings for guidance and cables for transferring power and information. These factors not only limit the stroke and functionality of the machine, but additionally disturb the motion of the mover, thereby reducing reliability, speed, and accuracy. This thesis focuses on the removal of these constraints and their effect on the system-level design of the machine. Three different contactless techniques can be incorporated into the motion system design to overcome the limitations of bearings and cables [23]. Hirano et al. (2017) used a musculoskeletal model and a Kinect TM based on a contactless motion analysis approach to measure muscle activation [24].

The proposed technique could be utilized in contactless transducer devices that can sense the vibrator parameters, especially where a small size is required. Harvested vibration energy is used for self-powered sensing by a Triboelectric Nanogenerator setup, especially for low-frequency vibrations [25]. Hadas et al. (2012) showed how to get energy from mechanical shocks by using a vibration energy harvester that is very sensitive [26].

\section{3- System Description and Analysis}

This study will show that there are many variants of this kind of mechanism. Some of these variants have advantages and disadvantages over others, which will be described by the simple mechanism and operation array design. The description and operation sequence for the proposed mechanism can be detailed as follows:

- The disk rotates in a $\mathrm{CCW}$ direction (by slightly starting moving or by rotating inertia of the previous cycle), and the rocker's magnet is near the disk, a repulsion force moves the most near (most force) magnet of the disk (edge magnet) away from the disk and accepts the oncoming towards the more far (less force) magnet disk as shown in Figure 2-a.

- The rotary disk (driven crank) continues the rotation, while the rotating force (repulsion force) is reduced, but enough to drive the connected load (see Figure 2-b).

- Once the angle of the disk reaches a point where the consequent magnets are far and far away and the starting (edge) magnet is near and near, the rocker arm must move away from the disk, as shown in Figure 2-c.

- Once the rocker magnet is far enough, the disk could continue rotating (by rotating inertia or by a concurrent shifted crank-rocker disk) without a strong repulsion resistance force. The disk could reach the point where the cycle starts again, as shown in Figure 2-d.

To identify exactly the parameters that create the motion and to determine the impact of these parameters on the power transmission, a mathematical analysis that describes the motion can model the system and be the tool of motion to identify the relations between the parameters. In this research, mathematical analysis will be focused on dimensional conditions that guarantee the continuity of motion transmission. However, dynamic analysis should be carried out after dimensional analysis and based on its conditions and results so that dynamic analysis can be part of future work. 


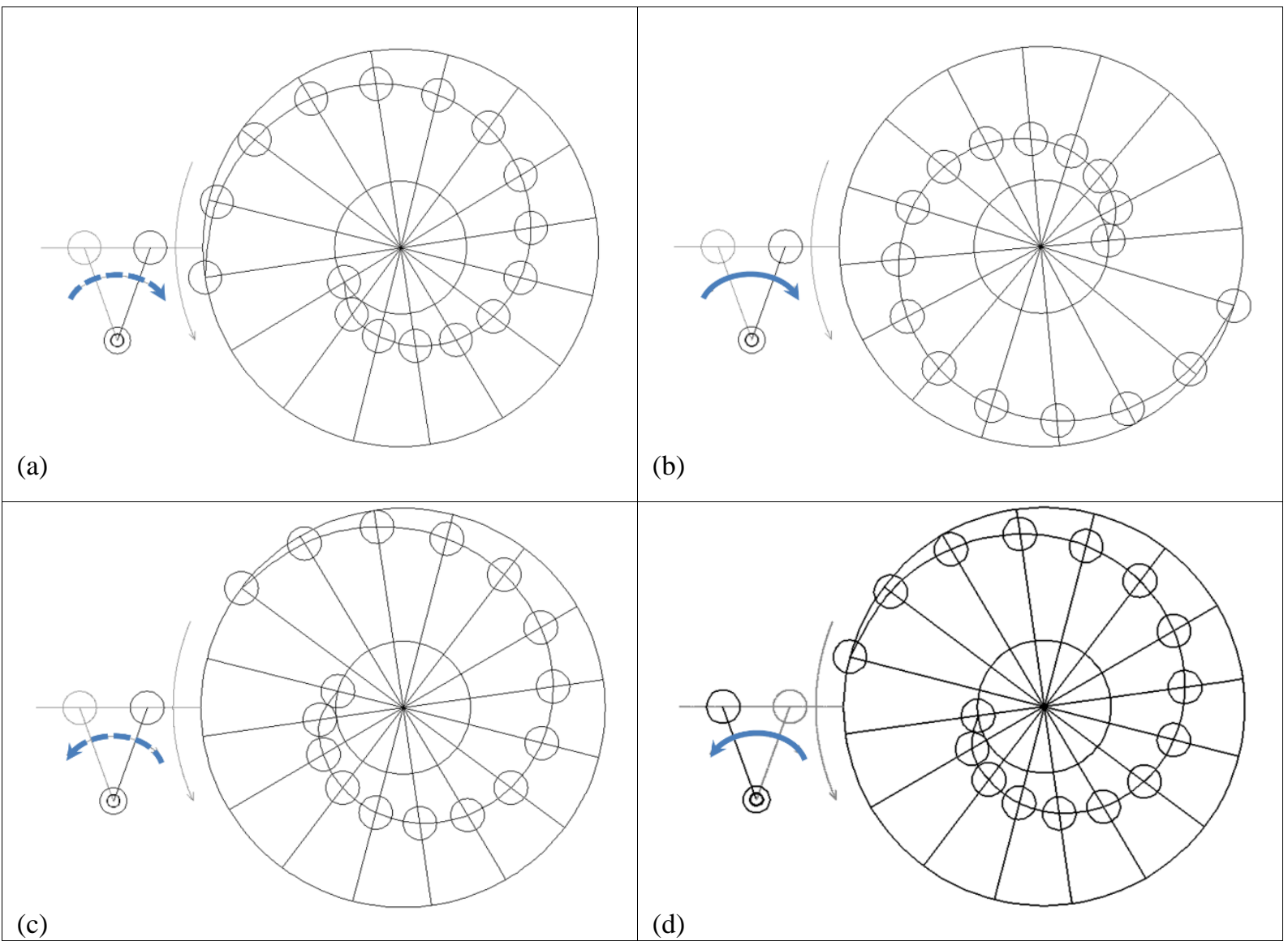

Figure 2. Sequential stages of the contactless crank-rocker machine operation; a: start-state of the cycle, b: middle-state of the cycle, $c$ : before end-state of the cycle, d: last-state of the cycle

\section{4- Results and Discussion}

Referring to Figure 3, which represents the basic structure of the proposed mechanism in this research, Considering that the rocker's magnet is $m_{r}$, while the most near two magnets of the disk is $m_{l}$ and $m_{2}$, the spaced angle between every two sequenced disk magnets are $a$, the disk radius is $R$, the inner dead radius is $r$, as the disk rotates, the distance $d$ increases, while the distance $d$ decreases. We will build the analysis based on $x$ value (radial magnet shift) a parameter design in terms of all other variable parameters, Figure 4 shows the model parameters of mathematical modelling.

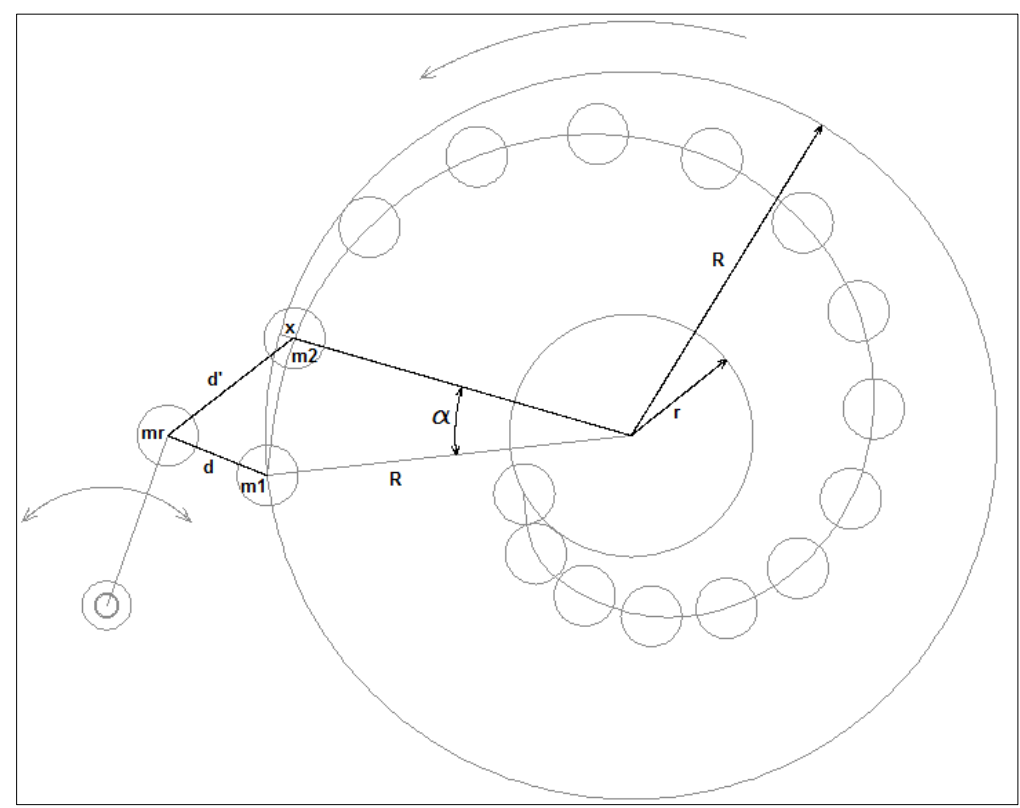

Figure 3. Parameter definition in contactless crank-rocker machine 


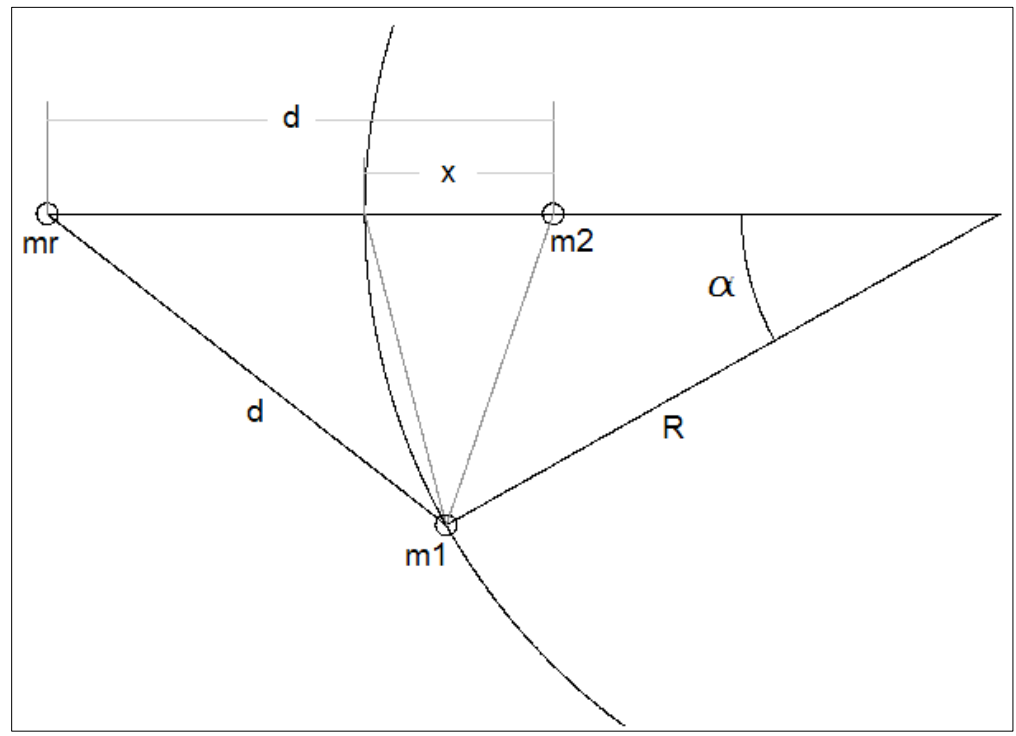

Figure 4. Model parameter in contactless crank-rocker machine

Considering the spaced angles are evenly the same by $\boldsymbol{N}$ number of angles, and the $\boldsymbol{x}$ shift values are also changing linearly along a complete one round, then:

$x_{k}=\left(\frac{N-k}{N}\right) \times(R-r), \quad k=[1,2,3, \ldots(N-1)]$

$k$ is the index of magnet on the disk, for the most edge magnet $m_{l}, k=N$, while it is for the next magnet $m_{l}, k=N-1$.

Since the magnets flux (values and direction) could be considered constant here, then the repulsion force depends on the distance between magnets only, and to simplify the analysis, we will consider the forces are only affected by the nearest two magnets from the rocker magnet (note that all other magnets fluxes are departed away by the unitarily of the magnets fields direction).

To make sure that the continuity and smoothness of movement, the repulsing force in between the rocker magnet and the nearest magnet on the disk should be always in the direction that results the same directional rotation of the disk. While the disk is rotating, and after the nearest magnet along the radial line (in between the center of the disk and the rocker magnet $m_{r}$ ) across this line and continuing the rotation, the next magnet on the disk should not be closer to rocker magnet than the last nearest magnet (i.e.: repulsion force along $d$ line should always be greater than repulsion force along $d$ ' in Figure 3). This could be done by shifting the next magnet toward the center of disk and parted from the rocker magnet by value of $x$.

The trade-off $x$ shifting value tolerated with the magnetic force, which affected significantly and exponentially by the distance in between two magnets, so that we have to calculate the optimized value of this shift, which should be as minimum as possible (best power transmission), while maintaining the continuity and smoothness condition which is described above. These calculations are depending on neglecting the inertia effect of rotating disk, which means that the rotating disk must be able to start rotating from any point without taking the condition of pre-rotating inertia. However, since the increasing in rotating inertia increases the smoothness of rotation, $x$ shifting value could be minimized and optimized considering of disk pre-rotation condition, these calculations could be retrieved through including dynamic analysis.

To find the value of $x$ that could be increased for the next sequential position of magnet, we will take the most critical case that happens after the nearest magnet $m_{l}$ along the radial line (in between the centre of the disk and the rocker magnet) across this line and continuing the rotation, and the next magnet on the disk $m_{2}$ be closer to rocker magnet $m_{r}$ than the last nearest magnet. This will happen when $m_{r}$ magnet being along $m_{2}$ at the same distance with $\mathrm{m}_{l}$ (i.e: when $d=d^{\prime}$ ) which is shown in Figure 4. By applying the general equation of triangular:

$$
\begin{aligned}
& d=\sqrt{\left(R^{2}+(R+d-x)^{2}\right)-2 R(R+d-x) \cos (\alpha)} \\
& d^{2}=R^{2}+R^{2}+R d-R x+R d+d^{2}-d x-R x-d x+x^{2}+2 R x \cos (\alpha)-2 R(R+d) \cos (\alpha) \\
& x^{2}+x(2 R \cos (\alpha)-2 R-2 d)=-2 R^{2}-2 R d+2 R^{2} \cos (\alpha)+2 R d(\cos (\alpha)-1) \\
& x^{2}+x(2 R \cos (\alpha)-2 R-2 d)=\left(2 R^{2}+2 R d\right)(\cos (\alpha)-1) \\
& x^{2}+x(2 R \cos (\alpha)-2 R-2 d)+\left(2 R^{2}+2 R d\right)(1-\cos (\alpha))=0
\end{aligned}
$$


By solving this 2nd order equation we will find:

$x=\frac{-(2 R \cos (\alpha)-2 R-2 d) \pm \sqrt{(2 R \cos (\alpha)-2 R-2 d)^{2}-4\left(2 R^{2}+2 R d\right)(1-\cos (\alpha))}}{2}$

Considering that $\boldsymbol{x}$ value cannot be more than R anyway, we can use one solution of $\boldsymbol{x}$ value that will give the minimum shift while the repulsing force in the same direction to continuously rotate the disk:

$$
x=(R+d-R \cos (\alpha))-\sqrt{\left.R^{2} \cos (\alpha)^{2}-R^{2}+d^{2}\right)}
$$

To retrieve the relation in between system parameters, and its relation with $\mathrm{x}$, we will use the Matlab tool to show the relation of each variable parameter on $x$ value, the following Matlab script code include the above equations, and finds the output results:

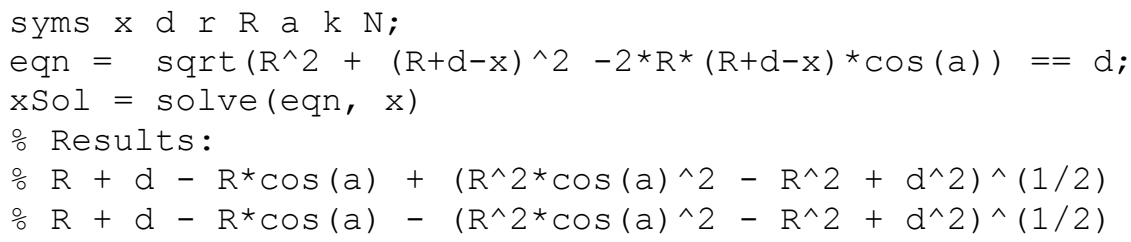

To find the effect of $d$ variation on $x$, the range of $d$ variation considered to be (from $4 \mathrm{~cm}$ to $6 \mathrm{~cm}$ ), while fixing all other parameters at $\left(R=10 \mathrm{~cm}, a=22.5^{\circ}\right)$ :

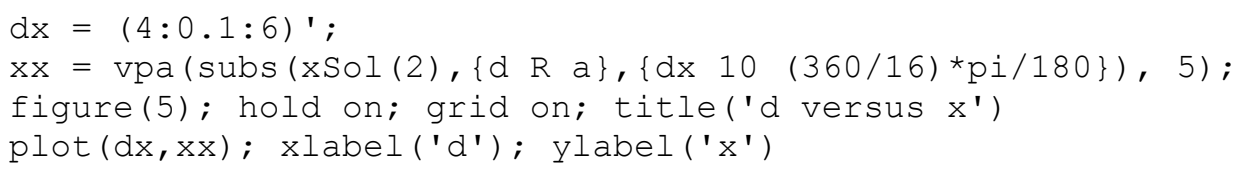

Shifting value $x$ versus $d$ relation is shown in Figure 5. To enhance the energy transfer, the repulsion force must be raised by reducing the distance $d$ in between $m_{r}$ and $m_{l}$ by reverse square relation [27], which means that $x$ shifting value will also be increased, that makes the repulsion force on the next magnet $m_{2}$ decreases; this concludes that a trade-off value of $d$ should be considered to maintain the best performance of energy transfer. Mainly, the distance $d$ value depends on the structure and setup of application. To find the effect of $R$ variation on $x$, the range of $R$ variation considered to be (from $8 \mathrm{~cm}$ to $12 \mathrm{~cm}$ ), while fixing all other parameters at $\left(d=5 \mathrm{~cm}, a=22.5^{\circ}\right)$ :
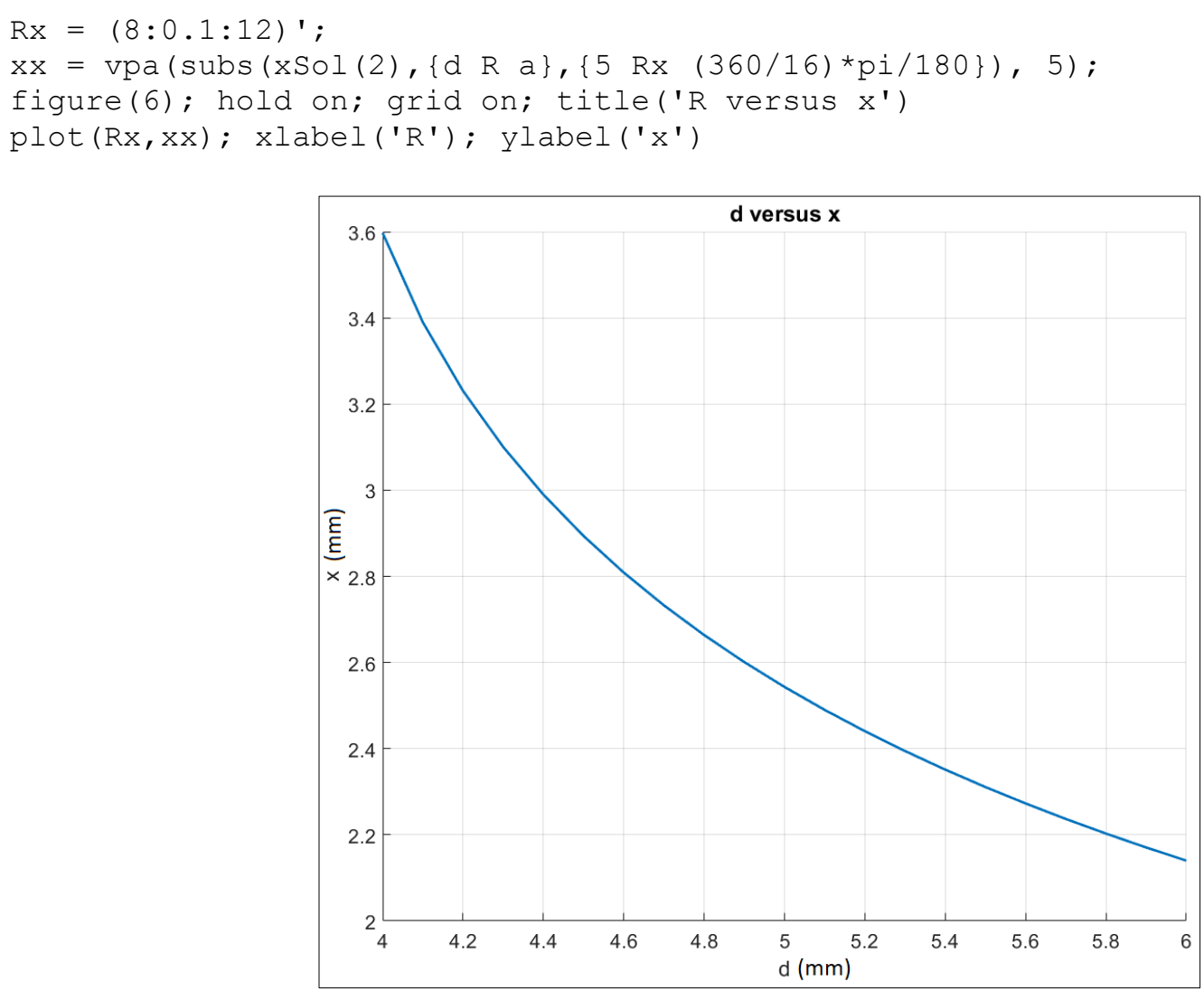

Figure 5. Shifting $x$ versus distance $d$ 
The $x$ versus $R$ relation is shown in Figure 6. As stated above, energy transfer will be improved by keeping $x$ value as small as possible, and this can be achieved by minimizing the disk radius $R$ which depends mainly on the structure and setup of application too.

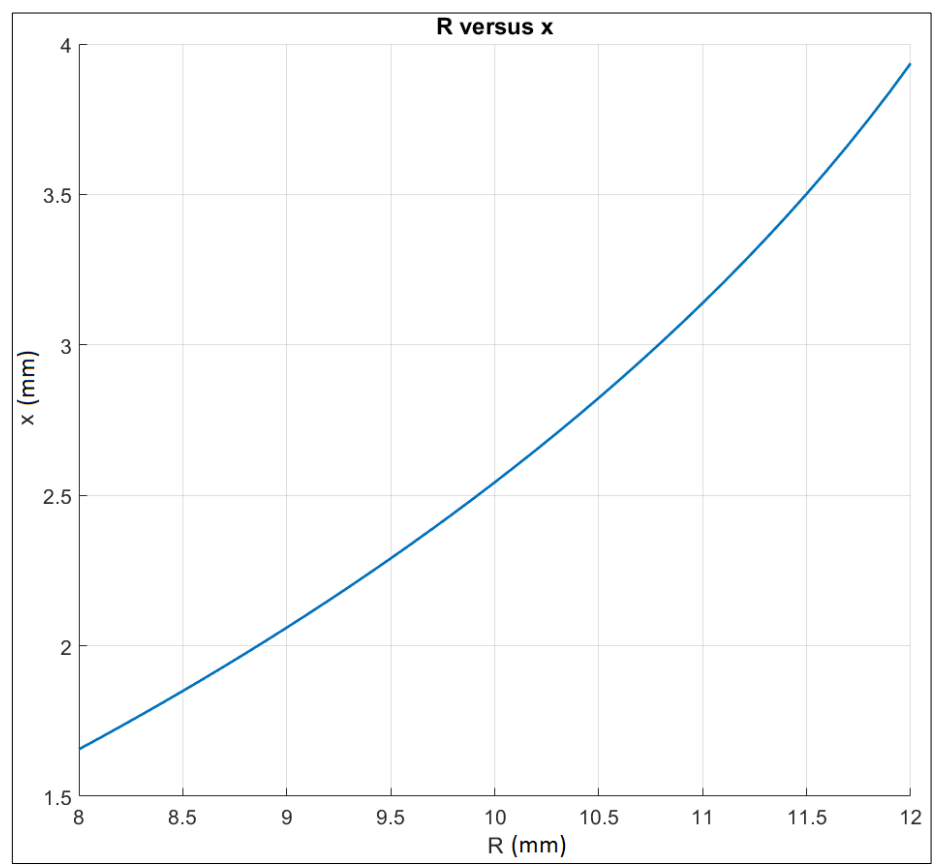

Figure 6. Shifting $\mathbf{x}$ versus disk radius $\mathbf{R}$

To find the effect of $N$ variation on $x$, the range of $N$ variation considered to be (from $14 \mathrm{~cm}$ to $24 \mathrm{~cm}$ ), which means $a$ variation (from $15^{\circ}$ to $\left.25.7^{\circ}\right)$, while fixing all other parameters at $(R=10 \mathrm{~cm}, d=5)$ :

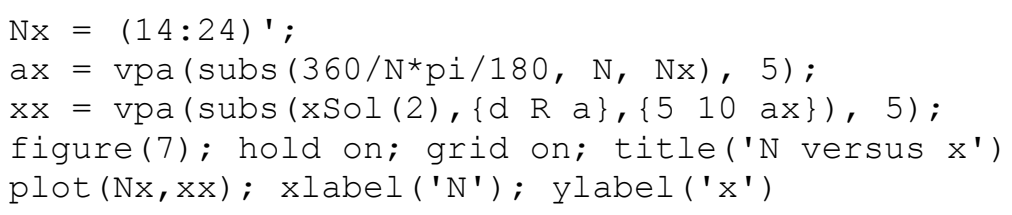

The $x$ versus $N$ relation shown in Figure 7, it shows that increasing the number of magnets on the rotary disk will decrease the shifting $x$, which improves the power transfer and its smoothness, it's noted that $N$ trade-offs with radius $R$.

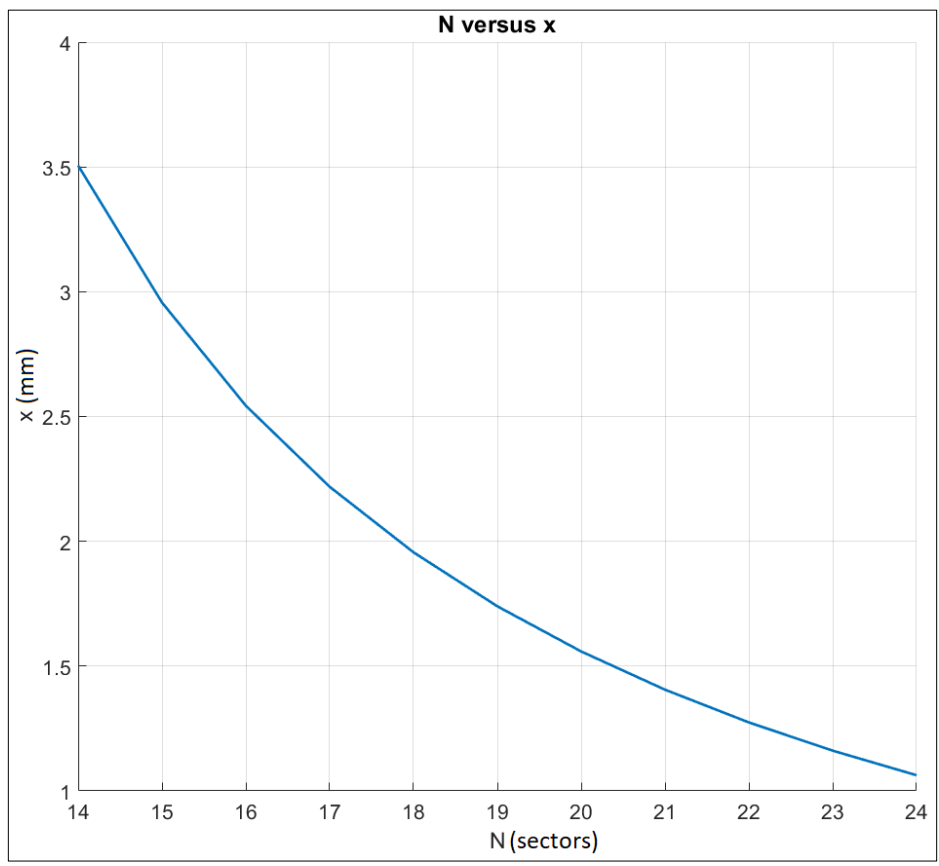

Figure 7. Shifting $\mathbf{x}$ versus number of rotating magnets $\mathbf{N}$ 
As shown in Figures 5 to 7, all dependent parameters are modelled against $\mathrm{x}$ parameter, and the designer of this mechanism can select the desired values that suite the related dimension in his application. To verify the operation of the proposed mechanism of contactless crank-rocker machine, and to illustrate the concept of motion, a simple model of proposed mechanism has been built and run, which approves the continuity of energy transmission using this technique. This model demonstrates the basic structure of this technique, and shows the sequence of operation, it was built based on the above analytical calculation of parameters.

Figure 8 shows a simple design of this model, the main part is a magnetic contact-less crank-rocker mechanism. It consists of a vibrating rocker with a permanent magnet fixed on its tip, and a rotating disk with distributed magnets fixed on it in the same manner described in Figure 1. This part transfers the input energy from the vibrating rocker to the rotating crank, which is the output energy (rotating load). All the other parts (electrical power supply feeding the electrical motor) are built to drive the rocker in vibrating motion and simulate the actual state of the vibrating energy source.

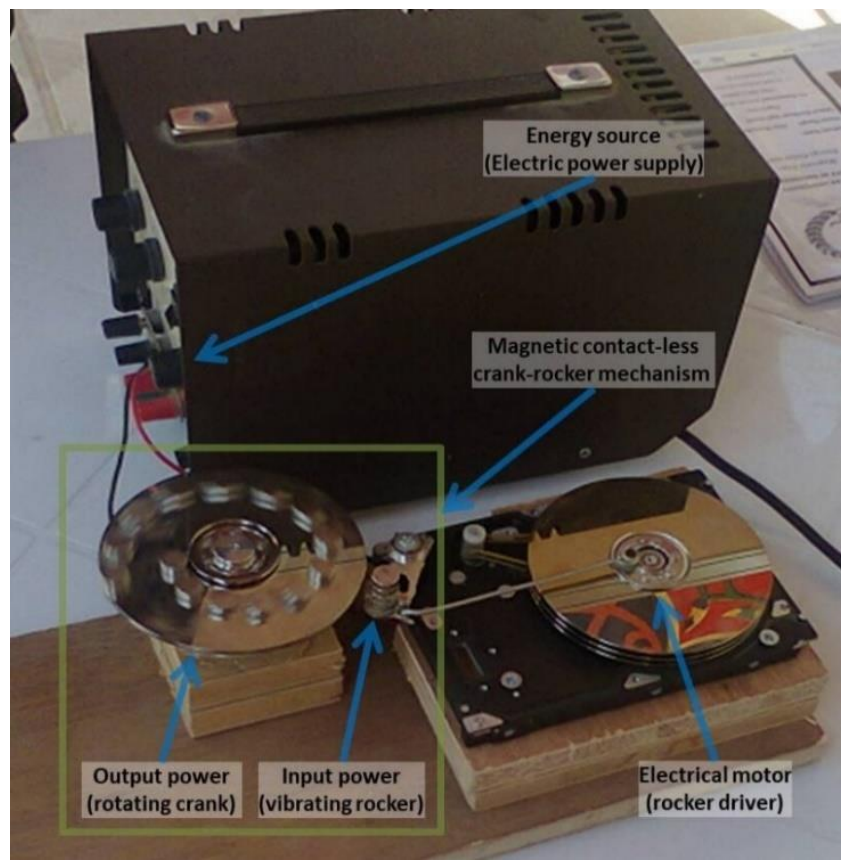

Figure 8. Simple demo structure of crank-rocker mechanism

As the provided technique in this paper is new, more researches and different designs could be applied on the same described principle of this technique. Also, the energy transmission action is performed through magnetic force, in which no direct connection in between energy source input and energy load output, several kinds of special applications can utilize this technique, where the isolation in between input and output energy stream is needed, and minimizing the friction loss of energy transmission process is required.

On the other hand, since this technique depends on magnet repulsion forces, the amount of transmitted energy is limited by dimensions of setup and strength of magnets, which concludes that this technique has more potential to be used in contact-less energy harvesting applications for small-scale of power, where collecting a limited amount of lost energy and converting it to useful energy is practical and useful. Also, MEMS could be another type of favorable area of application, where the micro magnet could be utilized to do special functions (as sensing and transducing), and where the isolation is highly preferable, and the miniature scale (small distance in between magnets) improves the magnetic transfer of energy. Depending on the principle of contactless crank rocker mechanism, different variants of design could be modified to match special applications and increase the efficiency of energy transferring; for instance, the sequence of magnets on rotary disk could be repeated as shown in Figure 9, this makes the average total shifting value $x$ to be minimized, and accepts higher alternating frequency of rocker.

Although different studies share some features with the provided technique in this work, and have similar final functions, no one contains the same architecture and process of work [28-30]. In this work, the energy transfers from the vibrating source to the rotating load in the mechanical type of energy, which has some advantages in specific applications and accepts the wideband frequency range of the vibrator. Most of available studies, the provided approach of transferring and deriving energy from vibrating source are proposed to convert it to electrical energy [29], a low-power controller IC has been fabricated using MEMS technology in order to convert ambient mechanical vibration into electrical energy to be used in powering low power electronic systems. One of the main limitations of converting energy of vibrator to electric energy is accepting the wideband frequency range of vibrator, especially when the mechanical 
vibration is low (as it is mostly available naturally in the surrounding), to overcome this limitation, some approaches have been proposed as described [30], it uses up-frequency converters to meet the high-frequency of piezoelectric generators.

To get the maximum energy transfer from the vibrator (rocker) to the rotating load (crank), the motion of the vibrator and rotating load must be synchronized, although the tuning approach for this synchronization is left to be studied in future work. A novel self-tuning concept of harvesting and converting vibration energy is presented by Gunn et al. (2021) [31]. By transferring energy through a variable distance of magnet force, a non-linearity will exist in energy flow, which effects the dynamic performance of the system. To minimize this non-linearity, the sequence of magnets on the rotary disk could be repeated as shown in Figure 9, and/or a multi-parallel mechanism of the rotating disk (crank) could be used simultaneously with equally-divided shifted angels of distributed magnets on the disk. In Zou et al. (2021) [32], a developed device capable of customizing nonlinear forces is provided. Yang et al. (2021) prepared a comprehensive review of harvesting nonlinear vibration energy and vibration suppression technology [33].

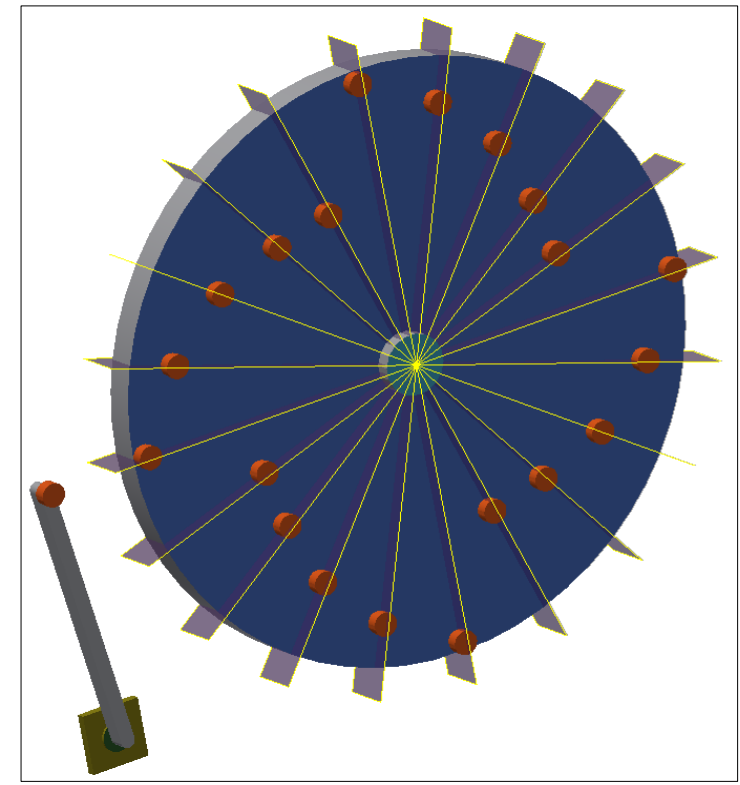

Figure 9. Repeated sequence variant of contactless magnets crank-rocker machine

\section{5- Conclusion}

A new proposed contactless crank-rocker machine mechanism that can convert vibrating mechanical motion into useful rotating motion without direct contact between the input and output streams of energy flow has been provided. The principle of operation and the structural parameters have been illustrated and analyzed. The mathematical analysis of dimensional parameters against design variables has been derived and analyzed. A simple demo model has been created to show, verify, and apply the derived relations and principles of work. This model approves the concept of the proposed technique and agrees with analytical calculations. In the future, and based on the provided dimensional analysis, a dynamic analysis of this mechanism and load synchronizing control could be provided. Based on the same basic design principle that is shown here, different designs of contactless crank-rocker machine mechanisms could be made, which could be used in different applications.

\section{6- Declarations}

\section{6-1- Author Contributions}

Conceptualization, A.I., and M.A.A.; methodology, A.I., and M.A.A.; software, A.I., and M.A.A; formal analysis, A.I., and M.A.A.; writing — original draft preparation, A.I., and M.A.A.; writing - review and editing, A.I., and M.A.A.; visualization, A.I., and M.A.A.; project administration, A.I., and M.A.A. All authors have read and agreed to the published version of the manuscript.

\section{6-2- Data Availability Statement}

The data presented in this study are available on request from the corresponding author.

\section{6-3- Funding}

The authors received no financial support for the research, authorship, and/or publication of this article. 


\section{6-4- Conflicts of Interest}

The authors declare that there is no conflict of interests regarding the publication of this manuscript. In addition, the ethical issues, including plagiarism, informed consent, misconduct, data fabrication and/or falsification, double publication and/or submission, and redundancies have been completely observed by the authors.

\section{7- References}

[1] Vaka, R., \& Keshri, R. K. (2017). Review on contactless power transfer for electric vehicle charging. Energies, $10(5), 636$. doi:10.3390/en10050636.

[2] Huang, L., Hu, A. P., Swain, A., Kim, S., \& Ren, Y. (2013). An overview of capacitively coupled power transfer - A new contactless power transfer solution. Proceedings of the 2013 IEEE 8th Conference on Industrial Electronics and Applications, ICIEA 2013, 461-465. doi:10.1109/ICIEA.2013.6566413.

[3] Sahai, A., \& Graham, D. (2011). Optical wireless power transmission at long wavelengths. 2011 International Conference on Space Optical Systems and Applications, ICSOS'11, 164-170. doi:10.1109/ICSOS.2011.5783662.

[4] Eves, E. E. (1992). Beamed Microwave Power Transmission and its Application to Space. IEEE Transactions on Microwave Theory and Techniques, 40(6), 1239-1250. doi:10.1109/22.141357.

[5] Norton, R.L. (2013). Kinematics and Dynamics of Machinery, Second edition, McGraw-Hill Higher Education, New York, USA.

[6] Razek, A. (2021). Review of contactless energy transfer concept applied to inductive power transfer systems in electric vehicles. Applied Sciences (Switzerland), 11(7), 3221. doi:10.3390/app11073221.

[7] Natesan, A. K. (1994). Kinematic analysis and synthesis of four-bar mechanisms for straight line coupler curves. Master thesis, Rochester Institute of Technology, New York, United States. Available online: https://scholarworks.rit.edu/theses/4658/ (accessed on December 2021).

[8] Niarchos, D. (2003). Magnetic MEMS: Key issues and some applications. Sensors and Actuators, A: Physical, 109(1-2), 166173. doi:10.1016/j.sna.2003.09.010.

[9] Fang, L. H., Hassan, S. I. S., Abd Rahim, R. Bin, \& Abd Malek, M. F. (2016). A study of vibration energy harvester. In ARPN Journal of Engineering and Applied Sciences 11(8), 5028-5041.

[10] Beeby, S. P., Tudor, M. J., \& White, N. M. (2006). Energy harvesting vibration sources for microsystems applications. Measurement Science and Technology, 17(12), 175- 195. doi:10.1088/0957-0233/17/12/R01.

[11] Kim, P., \& Seok, J. (2014). A multi-stable energy harvester: Dynamic modeling and bifurcation analysis. In Journal of Sound and Vibration, 333(21), 5525-5547. doi:10.1016/j.jsv.2014.05.054.

[12] Vidal, J. V., Turutin, A. V., Kubasov, I. V., Kislyuk, A. M., Kiselev, D. A., Malinkovich, M. D., Parkhomenko, Y. N., Kobeleva, S. P., Sobolev, N. A., \& Kholkin, A. L. (2020). Dual Vibration and Magnetic Energy Harvesting with Bidomain LiNbO3-Based Composite. IEEE Transactions on Ultrasonics, Ferroelectrics, and Frequency Control, 67(6), 1219-1229. doi:10.1109/TUFFC.2020.2967842.

[13] Fu, H., Theodossiades, S., Gunn, B., Abdallah, I., \& Chatzi, E. (2020). Ultra-low frequency energy harvesting using bi-stability and rotary-translational motion in a magnet-tethered oscillator. Nonlinear Dynamics, 101(4), 2131-2143. doi:10.1007/s11071020-05889-9.

[14] Tri Nguyen, H., Genov, D. A., \& Bardaweel, H. (2020). Vibration energy harvesting using magnetic spring based nonlinear oscillators: Design strategies and insights. Applied Energy, 269. doi:10.1016/j.apenergy.2020.115102.

[15] Redder, D. A. G., Brown, A. D., \& Andrew Skinner, J. (1999). A contactless electrical energy transmission system. IEEE Transactions on Industrial Electronics, 46(1), 23-30. doi:10.1109/41.744372.

[16] El-Refaie, A., Raminosoa, T., Reddy, P., Galioto, S., Pan, D., Grace, K., Alexander, J., \& Huh, K. K. (2017). Comparison of traction motors that reduce or eliminate rare-earth materials. IET Electrical Systems in Transportation, 7(3), $207-214$. doi:10.1049/iet-est.2016.0068.

[17] Woo, K. Il, Park, H. S., Cho, Y. H., \& Kim, K. H. (2005). Contactless energy transmission system for linear servo motor. IEEE Transactions on Magnetics, 41(5), 1596-1599. doi:10.1109/TMAG.2005.845025.

[18] Komiyama, H., \& Uchimura, Y. (2013). Contactless magnetic gear for robot control application. Electrical Engineering in Japan (English Translation of Denki Gakkai Ronbunshi), 184(4), 32-41. doi:10.1002/eej.22414.

[19] Tilli, A., Bosso, A., Conficoni, C., \& Hashemi, A. (2017). Integrated Control of Motion and Contactless Power Transfer for Doubly-Fed Induction Machines in Complex Rotary Apparatuses. IFAC-PapersOnLine, 50(1), 13129-13135. doi:10.1016/j.ifacol.2017.08.2166. 
[20] Mohammed, S. E., Baharom, M. B., Rashid Aziz, A. A., \& Zainal, E. Z. Z. (2019). Modelling of combustion characteristics of a single curved-cylinder spark-ignition crank-rocker engine. Energies, 12(17), 3313. doi:10.3390/en12173313.

[21] Koo, D. H., Hong, P. J., Cho, Y. H., \& Chung, K. S. (2002). Design and simulation of a contactless power transimission system. Automotive Electrical Equipment Optimization of Electronic Equipment, 377-382.

[22] Sang, Y., Karayaka, H. B., Yan, Y., Zhang, J. Z., Muljadi, E., \& Yu, Y. H. (2016). Energy extraction from a slider-crank wave energy converter under irregular wave conditions. Oceans 2015, 1-7. MTS/IEEE Washington, United States. doi:10.23919/oceans.2015.7401873.

[23] Boeij, de, J. (2009). Multi-level contactless motion system. Technische Universiteit Eindhoven. Dissertations, Doctor of Philosophy, Eindhoven, Netherlands. doi:10.6100/IR640039.

[24] Hirano, Y., Kushida, D., \& Matsumoto, H. (2018). Contactless motion analysis system using a kinect and musculoskeletal model. 2017 IEEE Life Sciences Conference, LSC 2017, 2018-January, 308-311. doi:10.1109/LSC.2017.8268204.

[25] Chen, J., \& Wang, Z. L. (2017). Reviving Vibration Energy Harvesting and Self-Powered Sensing by a Triboelectric Nanogenerator. Joule, 1(3), 480-521. doi:10.1016/j.joule.2017.09.004.

[26] Hadas, Z., Vetiska, V., Singule, V., Andrs, O., Kovar, J., \& Vetiska, J. (2012). Energy harvesting from mechanical shocks using a sensitive vibration energy harvester. International Journal of Advanced Robotic Systems, 9. doi:10.5772/53948.

[27] Kodama, K. (2009). A simple demonstration of a general rule for the variation of magnetic field with distance. Physics Education, 44(3), 276-280. doi:10.1088/0031-9120/44/3/007.

[28] Rantz, R., \& Roundy, S. (2019). Characterization of Real-world Vibration Sources and Application to Nonlinear Vibration Energy Harvesters. Energy Harvesting and Systems, 4(2), 67-76. doi:10.1515/ehs-2016-0021.

[29] Meninger, S., Mur-Miranda, J. O., Amirtharajah, R., Chandrakasan, A. P., \& Lang, J. H. (2001). Vibration-to-electric energy conversion. IEEE Transactions on Very Large Scale Integration (VLSI) Systems, 9(1), 64-76. doi:10.1109/92.920820.

[30] Dauksevicius, R., Briand, D., Lockhart, R., Vásquez Quintero, A., De Rooij, N., Gaidys, R., \& Ostasevicius, V. (2014). Frequency up-converting vibration energy harvester with multiple impacting beams for enhanced wideband operation at low frequencies. In Procedia Engineering, 87, 1517-1520. doi:10.1016/j.proeng.2014.11.587.

[31] Gunn, B., Alevras, P., Flint, J. A., Fu, H., Rothberg, S. J., \& Theodossiades, S. (2021). A self-tuned rotational vibration energy harvester for self-powered wireless sensing in powertrains. Applied Energy, 302. doi:10.1016/j.apenergy.2021.117479.

[32] Zou, D., Liu, G., Rao, Z., Tan, T., Zhang, W., \& Liao, W. H. (2021). A device capable of customizing nonlinear forces for vibration energy harvesting, vibration isolation, and nonlinear energy sink. Mechanical Systems and Signal Processing, 147. doi:10.1016/j.ymssp.2020.107101.

[33] Yang, T., Zhou, S., Fang, S., Qin, W., \& Inman, D. J. (2021). Nonlinear vibration energy harvesting and vibration suppression technologies: Designs, analysis, and applications. Applied Physics Reviews, 8(3), 31317. doi:10.1063/5.0051432. 